International Mathematical Forum, 1, 2006, no. 29, 1405 - 1422

\title{
SPECTRAL AND AFFINE FRACTAL METHODS IN SIGNAL PROCESSING
}

\author{
M.A. Navascués \\ Dept. of Applied Mathematics. \\ Engineering Polytechnic Center. \\ University of Zaragoza. Spain. \\ manavas@unizar.es \\ http://pcmap.unizar.es/ navascues/ \\ M.V. Sebastián \\ Dept. of Mathematics. \\ Engineering Polytechnic Center. \\ University of Zaragoza. Spain. \\ msebasti@unizar.es
}

\begin{abstract}
Fourier expansions display the spectral content of a signal and provide explicit representations in time and frequency domains. In this paper, we define trigonometric approximants of a real sampled signal by means of fractal techniques. The construction allows us to obtain, under some hypotheses, exact relations between the discrete power spectrum of a signal and its fractal dimension. In the last part of the paper, an electroencephalographic application is described.
\end{abstract}

Mathematics Subject Classification: 28A80, 42A10, 65D05, 37M10.

Keywords: Fractal interpolation functions, discrete power spectrum, fractal dimension.

\section{Introduction}

In past decades, some questions arose in the bosom of the scientific community related to the character of deterministic or stochastic of some experimental signals, for instance electroencephalographic recordings (EEG). Obviating such a discussion, it is clear that the methodology of dynamical systems and that 
of fractal sets in particular, has proved to be useful for the modelization and quantification of experimental data.

Until the seventies, the EEG quantification was only performed by means of the FFT algorithm. However, the signal recorded depends on the reference chosen and this fact makes it difficult to compare results of experiments carried out by different teams and equipments. It is well known also that in many pathologies as, for instance, the disorders related with learning, the FFT study is not conclusive. It is convenient, on one hand, to refine the techniques of recording and, on the other, to complement the spectral study with other numerical methods.

The dynamical approach of bioelectric recordings assumes that the signal can be modelized by different kinds of attractors. These sets may be discriminated estimating several parameters as, for instance, fractal dimensions. The computation of the Rényi spectrum has been used since the eighties as a numerical tool and many relations have been found linking: dimensions and epilepsy [1], dimensions and sleep [15]-[16], dimensions and intelligence quotient [10], dimensions and schizophrenics [5], etc.

We have proposed, in reference [14], a technique of fitting and reconstructing of any signal by means of fractal interpolation functions [2]. The fractal dimension is computed easily by means of explicit formulae involving the time samples. The proposed quantification allows us to assign numerical values to an EEG recording in order to discriminate between different brain stages or to correlate it with external variables. The parameters do not require any hypothesis of stationarity of the signal nor long segments of recordings. This kind of procedures provides computational agility in order to analyse so much segments as desired, and the possibility of studying the evolution of the fractal dimension in a specific process, mental task or pathology, to stablish timevarying properties of the EEG trace. The computation of fractal dimensions for different brain states and many preliminary results of its application to clinical processes suggest that it contains the basic numerical description of the neurophysiologic states.

Several authors have inquired into the relations existing among different methods of EEG quantification: nonlinear parameters arised from chaos theory, FFT and Hjorth parameters. For example, in the reference [20], a comparative study between nonlinear indices and Hjorth methods by means of factor analysis is performed. They obtain a high statistical correlation between an index of mean frequency of the EEG and fractal dimension.

One of the aims of the present study is to prove exact relations between fractal dimensions and spectral powers. The proof confirms that fractal quantifiers can also be used for the exploration of spectral properties of experimental recordings. Fractal dimension allows us to represent the EEG signal in time and frequency domains since the complete spectrum of the signal and the au- 
tocorrelation function can be retrieved from it. All the particular spectral powers can be computed with any desired degree of approximation. The reconstruction of the power spectrum from the fractal dimension justifies its use as objective quantifier of the EEG signal, obviating the discussion about the description of intrinsic properties of specific neurophysiologic processes.

\section{Discrete power spectrum}

Let $L^{2}(T)$ be the Hilbert space of functions of period $T>0$ with integrable square in an interval $I$ of length $T$, provided with the inner product:

$$
<f, g>_{L^{2}(T)}=\frac{1}{T} \int_{I} f(t) g^{*}(t) d t
$$

where $g^{*}$ is the complex conjugate of $g$. The associated norm is:

$$
\|f\|_{L^{2}(T)}^{2}=<f, f>_{L^{2}(T)}=\frac{1}{T} \int_{I}|f(t)|^{2} d t
$$

Let us consider $w_{0}=2 \pi / T$ and $\varphi_{n}(t)=e^{i n w_{0} t}$ for $n \in Z$. The family $\left\{\varphi_{n}(t)\right\}_{n \in Z}$ is an orthonormal system of $L^{2}(T)$ respect to previous inner product, in fact is a basis of Hilbert for this space. If $x(t) \in L^{2}(T)$, the Fourier coefficients of $x$ are defined as

$$
c_{n}=<x, \varphi_{n}>_{L^{2}(T)}=\frac{1}{T} \int_{I} x(t) e^{-i n w_{0} t} d t
$$

The Fourier series $S_{\infty}=\sum_{n=-\infty}^{+\infty} c_{n} \varphi_{n}$ is convergent in $L^{2}(T)$ and its sum is $x$, that is to say

$$
x(t)=\sum_{n=-\infty}^{+\infty} c_{n} e^{i n w_{0} t}
$$

in $L^{2}$ sense. Due to the orthogonality of the functions $\varphi_{n}$, the finite Fourier sum (obtained by truncation of the series) constitutes the best approximation of $x(t)$ for a given order (in terms of trigonometric polynomials). In addition, the following equalities are verified (Theorem of Parseval):

$$
\|x\|_{L^{2}(T)}^{2}=\frac{1}{T} \int_{I}|x(t)|^{2} d t=\sum_{n=-\infty}^{+\infty}\left|c_{n}\right|^{2}<+\infty
$$

and if $x(t), y(t) \in L^{2}(T)$

$$
<x, y>=\frac{1}{T} \int_{I} x(t) y^{*}(t) d t=\sum_{n=-\infty}^{+\infty} c_{n}^{x}\left(c_{n}^{y}\right)^{*}
$$


where $c_{n}^{x}$ and $c_{n}^{y}$ are the Fourier coefficients of $x(t)$ and $y(t)$ respectively [18]. The collection of powers $\left|c_{n}\right|^{2}$ is usually called Discrete Power Spectrum of the signal $x(t)$. The average autocorrelation function of $x(t) \in L^{2}(T)$ is defined by:

$$
R(\tau)=\frac{1}{T} \int_{I} x(t-\tau) x(t) d t
$$

The equality (4) along with the Fourier series of $x(t-\tau)(2)$ :

$$
x(t-\tau)=\sum_{n=-\infty}^{+\infty} c_{n} e^{-i n w_{0} \tau} e^{i n w_{0} t}
$$

allows us to compute $R(\tau)$ in terms of the Fourier coefficients of the real signal $x(t)$ :

$$
R(\tau)=\frac{1}{T} \int_{I} x(t) x(t-\tau) d t=\sum_{n=-\infty}^{+\infty} c_{n} c_{n}^{*} e^{i n w_{0} \tau}
$$

and

$$
R(\tau)=\sum_{n=-\infty}^{+\infty}\left|c_{n}\right|^{2} e^{i n w_{0} \tau}
$$

where $w_{0}=2 \pi / T$. The spectral powers $\left|c_{n}\right|^{2}$ are then the Fourier coefficients of $R(\tau)$. The autocorrelation function provides the information obtained from a conventional frequency analysis, where the phase information is omitted. Besides, the discrete power spectrum allows us to obtain the mean power (or energy content) of a signal

$$
E=\frac{1}{T} \int_{I}|x(t)|^{2} d t=\sum_{n=-\infty}^{+\infty}\left|c_{n}\right|^{2}
$$

\section{Affine fractal interpolation functions}

In this work we reconstruct the discrete power spectrum of an experimental sampled signal by means of fractal interpolation (see references [2], [3], [13]) and we express it in terms of the fractal dimension.

Let $t_{0}<t_{1}<\ldots<t_{N}$ be real numbers, and $I=\left[t_{0}, t_{N}\right]$ the closed interval that contains them. Let a set of data points $\left\{\left(t_{n}, x_{n}\right) \in I \times R: n=\right.$ $0,1,2, \ldots, N\}$ be given. Set $I_{n}=\left[t_{n-1}, t_{n}\right]$ and let $L_{n}: I \rightarrow I_{n}, n \in\{1,2, \ldots, N\}$ be contractive homeomorphisms such that:

$$
L_{n}\left(t_{0}\right)=t_{n-1}, L_{n}\left(t_{N}\right)=t_{n}
$$




$$
\left|L_{n}\left(c_{1}\right)-L_{n}\left(c_{2}\right)\right| \leq l\left|c_{1}-c_{2}\right| \quad \forall c_{1}, c_{2} \in I
$$

for some $0 \leq l<1$.

Let $-1<\alpha_{n}<1 ; n=1,2, \ldots, N, F=I \times[c, d]$ for some $-\infty<c<d<$ $+\infty$ and $N$ continuous mappings, $F_{n}: F \rightarrow R$ be given satisfying:

$$
F_{n}\left(t_{0}, x_{0}\right)=x_{n-1}, \quad F_{n}\left(t_{N}, x_{N}\right)=x_{n}
$$

where $n=1,2, \ldots, N$.

$$
\left|F_{n}(t, x)-F_{n}(t, y)\right| \leq \alpha_{n}|x-y|
$$

with $t \in I$, and $x, y \in R$.

Now define functions

$$
w_{n}(t, x)=\left(L_{n}(t), F_{n}(t, x)\right)
$$

$\forall n=1,2, \ldots, N$.

Theorem 3.1. [3, 2]: The iterated function system (IFS) $\left\{F, w_{n}\right.$ : $n=1,2, \ldots, N\}$ defined above admits a unique attractor $G . G$ is the graph of a continuous function $f: I \rightarrow R$ which obeys $f\left(t_{n}\right)=x_{n}$ for $n=0,1,2, \ldots, N$.

The previous function is called a fractal interpolation function (FIF) corresponding to $\left\{\left(L_{n}(t), F_{n}(t, x)\right)\right\}_{n=1}^{N}$.

Let $\mathcal{G}$ be the set of continuous functions $f:\left[t_{0}, t_{N}\right] \rightarrow[c, d]$ such that $f\left(t_{0}\right)=x_{0} ; f\left(t_{N}\right)=x_{N} \cdot \mathcal{G}$ is a complete metric space respect to the uniform norm. Define a mapping $T: \mathcal{G} \rightarrow \mathcal{G}$ by:

$$
(T f)(t)=F_{n}\left(L_{n}^{-1}(t), f \circ L_{n}^{-1}(t)\right)
$$

$\forall t \in\left[t_{n-1}, t_{n}\right], \quad n=1,2, \ldots, N$.

$T$ is a contraction mapping on the metric space $\left(\mathcal{G},\|\cdot\|_{\infty}\right)$ :

$$
\|T f-T g\|_{\infty} \leq|\alpha|_{\infty}\|f-g\|_{\infty}
$$

where $|\alpha|_{\infty}=\max \left\{\left|\alpha_{n}\right| ; n=1,2, \ldots, N\right\}$. Since $|\alpha|_{\infty}<1, T$ possesses a unique fixed point on $\mathcal{G}$, that is to say, there is $f \in \mathcal{G}$ such that $(T f)(t)=$ $f(t) \forall t \in\left[t_{0}, t_{N}\right]$. This function is the FIF corresponding to $w_{n}$ and it is the unique $f \in \mathcal{G}$ satisfying the functional equation $[3,2]$ :

$$
f(t)=F_{n}\left(L_{n}^{-1}(t), f \circ L_{n}^{-1}(t)\right)
$$


$n=1,2, \ldots, N, \quad t \in I_{n}=\left[t_{n-1}, t_{n}\right]$, that is to say

$$
f(t)=\alpha_{n} f \circ L_{n}^{-1}(t)+q_{n} \circ L_{n}^{-1}(t)
$$

The most widely studied fractal interpolation functions so far are defined by the IFS

$$
\left\{\begin{array}{l}
L_{n}(t)=a_{n} t+b_{n} \\
F_{n}(t, x)=\alpha_{n} x+q_{n}(t)
\end{array}\right.
$$

where

$$
a_{n}=\frac{\left(t_{n}-t_{n-1}\right)}{\left(t_{N}-t_{0}\right)} \quad \text { and } \quad b_{n}=\frac{\left(t_{N} t_{n-1}-t_{0} t_{n}\right)}{\left(t_{N}-t_{0}\right)}
$$

$\alpha_{n}$ is called a vertical scaling factor of the transformation $w_{n}$ and $\alpha=\left(\alpha_{1}, \alpha_{2}, \ldots, \alpha_{N}\right)$ is the scale vector of the IFS. If $q_{n}(t)$ is a line, the FIF is termed affine. In this case, by $(10), q_{n}(t)=q_{n 1} t+q_{n 0}$, where

$$
\begin{gathered}
q_{n 1}=\frac{x_{n}-x_{n-1}}{t_{N}-t_{0}}-\alpha_{n} \frac{x_{N}-x_{0}}{t_{N}-t_{0}} \\
q_{n 0}=\frac{t_{N} x_{n-1}-t_{0} x_{n}}{t_{N}-t_{0}}-\alpha_{n} \frac{t_{N} x_{0}-t_{0} x_{N}}{t_{N}-t_{0}}
\end{gathered}
$$

According to Theorem of Barnsley [2] the fractal dimension $D$ of the graph of an affine fractal interpolation function (AFIF) satisfies the equation:

$$
\sum_{n=1}^{N}\left|\alpha_{n}\right| a_{n}^{D-1}=1
$$

where $\alpha_{n}$ is the $n$ - th vertical scaling factor of the IFS and $a_{n}$ the coefficients defined in (15). If the nodes are equidistant, $a_{n}=1 / N$ and

$$
D=1+\frac{\log \left(\sum_{n=1}^{N}\left|\alpha_{n}\right|\right)}{\log N}
$$

This formula for the dimension is valid in the case $\sum_{n=1}^{N}\left|\alpha_{n}\right|>1$. Otherwise, the fractal dimension is one [3]. The parameter lies between 1 and 2. Its computation is simple, the use of delay variables is not necessary and there are no problems of convergence or insufficient number of points.

If the scaling factors are positive and do not depend on the subinterval, from (19)

$$
\alpha=N^{D-2}
$$

From here on, we consider an interval $I$ of length $T$ and $x_{0}=x_{N}$, in order to handle functions of $L^{2}(T)$. 


\section{Discrete power spectrum versus dimension}

In this paragraph we obtain, under some hypotheses, exact formulae of the spectral powers $\left|c_{n}\right|^{2}$ in terms of the fractal dimension $D$.

Let $f(t)$ be a real function, $f(t) \in L^{2}(T)$, from (1)

$$
\left|c_{n}\right|^{2}=\frac{1}{T^{2}}\left[\left(\int_{I} f(t) \cos \left(n w_{0} t\right) d t\right)^{2}+\left(\int_{I} f(t) \sin \left(n w_{0} t\right) d t\right)^{2}\right]
$$

and

$$
\begin{gathered}
\left|c_{n}\right|^{2}=\frac{1}{T^{2}}\left[\left(\int_{I} f(t)\left(\sum_{p=0}^{+\infty} \frac{\left(n w_{0} t\right)^{2 p}}{(2 p) !}(-1)^{p}\right) d t\right)^{2}+\right. \\
\left.\left(\int_{I} f(t)\left(\sum_{p=0}^{+\infty} \frac{\left(n w_{0} t\right)^{2 p+1}}{(2 p+1) !}(-1)^{p}\right) d t\right)^{2}\right] \\
\left|c_{n}\right|^{2}=\frac{1}{T^{2}}\left[\left(\sum_{p=0}^{+\infty}(-1)^{p} \frac{\left(n w_{0}\right)^{2 p}}{(2 p) !} M_{2 p}\right)^{2}+\left(\sum_{p=0}^{+\infty}(-1)^{p} \frac{\left(n w_{0}\right)^{2 p+1}}{(2 p+1) !} M_{2 p+1}\right)^{2}\right]
\end{gathered}
$$

where

$$
M_{m}=\int_{I} t^{m} f(t) d t
$$

In the reference [3], Barnsley deduces a recurrent expression to compute the integral moments $M_{m}$ for an AFIF.

The formula is given by

$$
M_{m}=\frac{1}{\left(1-\sum_{n=1}^{N} a_{n}^{m+1} \alpha_{n}\right)}\left(\sum_{k=0}^{m-1}\left(\begin{array}{c}
m \\
k
\end{array}\right) M_{k} \sum_{n=1}^{N} a_{n}^{k+1} \alpha_{n} b_{n}^{m-k}+Q_{m}\right)
$$

where

$$
Q_{m}=\int_{I} t^{m} Q(t) d t
$$

and

$$
Q(t)=q_{n} \circ L_{n}^{-1}(t) \quad \text { if } \quad t \in I_{n}
$$

For an AFIF, it is easy to deduce (see the details in [17]) that $\forall t \in I_{n}$ :

$$
q_{n} \circ L_{n}^{-1}(t)=f_{0}(t)-\alpha_{n} r \circ L_{n}^{-1}(t)
$$


where $f_{0}$ is a piecewise linear function with vertices on the data $\left\{\left(t_{n}, x_{n}\right)\right\}_{n=0}^{N}$ and $r(t)$ is the line joining $\left(t_{0}, x_{0}\right)$ and $\left(t_{N}, x_{N}\right)$ :

$$
r(t)=\frac{x_{N}-x_{0}}{t_{N}-t_{0}} t+\frac{t_{N} x_{0}-t_{0} x_{N}}{t_{N}-t_{0}}
$$

In this case, $x_{0}=x_{N}$ and $r(t)=x_{0} \forall t \in I$. If the scaling factors are constant, $\forall t \in I$

$$
Q(t)=f_{0}(t)-\alpha x_{0}
$$

and the computation of $Q_{m}$ is straightforward from (24). If the nodes are equidistant

$$
a_{n}=\frac{1}{N}
$$

and, considering constant positive scaling factors, from (20) we obtain $M_{m}$ in terms of the fractal dimension $D$

$$
M_{m}=\frac{1}{1-N^{D-2-m}}\left(\sum_{k=0}^{m-1}\left(\begin{array}{c}
m \\
k
\end{array}\right) M_{k} N^{D-k-3} \sum_{n=1}^{N} b_{n}^{m-k}+Q_{m}\right)
$$

The moment $M_{0}$ can be computed using (13)

$$
\begin{gathered}
M_{0}=\int_{I} f(t) d t=\sum_{n=1}^{N} \int_{I_{n}}\left(\alpha_{n} f \circ L_{n}^{-1}(t)+q_{n} \circ L_{n}^{-1}(t)\right) d t \\
M_{0}=\alpha \sum_{n=1}^{N} \int_{I_{n}} f \circ L_{n}^{-1}(t) d t+Q_{0}
\end{gathered}
$$

with the change $L_{n}^{-1}(t)=\tilde{t}$

$$
M_{0}=\alpha M_{0}+Q_{0}
$$

and

$$
M_{0}=\frac{Q_{0}}{1-\alpha}=\frac{Q_{0}}{1-N^{D-2}}
$$

From the moments $M_{m}$ we can obtain the discrete power spectrum and therefrom the mean power (7) and the autocorrelation function of the signal (6). 


\section{$5 \quad$ Finite real sum of Fourier}

From here on we assume, without loss of generality, that $T=2 \pi$ in order to simplify the calculus. We consider the space of $2 \pi$-periodic continuous functions

$$
\mathcal{C}(2 \pi)=\{f:[-\pi, \pi] \rightarrow R ; \text { continuous, } f(-\pi)=f(\pi)\}
$$

and

$$
\begin{gathered}
\mathcal{S}_{m}: \mathcal{C}(2 \pi) \rightarrow \mathcal{C}(2 \pi) \\
f \rightarrow S_{m}(t)
\end{gathered}
$$

where $S_{m}(t)$ is the Fourier finite real sum of $f$ defined by

$$
S_{m}(t)=\sum_{n=0}^{m}\left(a_{n} \cos \left(n w_{0} t\right)+b_{n} \sin \left(n w_{0} t\right)\right)
$$

$a_{n}=c_{n}+c_{-n}$, and $b_{n}=i\left(c_{n}-c_{-n}\right)(1)$.

$\mathcal{S}_{m}$ is a linear and bounded operator, and the following inequality holds [4]

$$
\left\|\mathcal{S}_{m}\right\| \leq 3+\log (m)
$$

In addition $\mathcal{S}_{m}$ is a projection, $\mathcal{S}_{m} \circ \mathcal{S}_{m}=\mathcal{S}_{m}$.

The Fourier sums show the spectral content of a signal and provide, this way, an explicit representation in the domains of time and frequency. This fact is particularly important in the case of the FIF, as they do not have an explicit expression. Fourier expansions make the filtering and processing of a signal easier, omitting or retrieving frequencies in a given band.

In principle a Fourier finite sum only assures a good representation of the function in $L^{2}$ sense, according to paragraph 2 . In the following we deduce some results in order to bound the uniform error committed in the approximation of the function by a finite sum.

In order to discriminate the error committed, we will use the following results.

Proposition 5.1. If $f$ is an AFIF with data points $\left\{\left(t_{n}, x_{n}\right)\right\}_{n=0}^{N}$ and $f_{0}$ is a piecewise linear and continuous function, with vertices on the data, then,

$$
\left\|f-f_{0}\right\|_{\infty} \leq \frac{2|\alpha|_{\infty}}{1-|\alpha|_{\infty}} X_{\max }
$$

where $X_{\max }=\max _{0 \leq n \leq N}\left\{\left|x_{n}\right|\right\}$. 
Proof. See reference [14].

Proposition 5.2. Let $f$ and $f_{0}$ be as in Proposition 5.1 and $f, f_{0} \in \mathcal{C}(2 \pi)$, the following inequality holds

$$
\left\|\mathcal{S}_{m} f-\mathcal{S}_{m} f_{0}\right\|_{\infty} \leq(3+\log m) \frac{2|\alpha|_{\infty}}{1-|\alpha|_{\infty}} X_{\max }
$$

where $X_{\max }=\max _{0 \leq n \leq N}\left\{\left|x_{n}\right|\right\}$.

Proof. It is a Consequence of the linearity of $\mathcal{S}_{m}$, the inequality (30) and Proposition 5.1.

Lemma 5.3. Let $f_{0}$ be a piecewise linear and continuous function with vertices $\left\{\left(t_{n}, x_{n}\right)\right\}_{n=0}^{N}, t_{n}-t_{n-1}=h$ constant and $t_{0}=-\pi, t_{N}=\pi$. For $m$ sufficiently large $(m \geq N / 2)$,

$$
w\left(\frac{\pi}{m}\right) \leq \frac{N \Delta_{x}}{m}
$$

with $w(\delta)$ being the modulus of continuity of $f_{0}$ and $\Delta_{x}=\max _{1 \leq n \leq N}\left\{\mid x_{n}-\right.$ $\left.x_{n-1} \mid\right\}$.

Proof. The modulus of continuity of $f_{0}$ is defined by

$$
w(\delta)=\sup _{\left|t-t^{\prime}\right| \leq \delta}\left|f_{0}(t)-f_{0}\left(t^{\prime}\right)\right|
$$

Let $t, t^{\prime}$ be such that $\left|t-t^{\prime}\right| \leq \frac{\pi}{m}$. For $m$ sufficiently large $\frac{\pi}{m} \leq h=\frac{2 \pi}{N}$, $(m \geq N / 2)$. If $t, t^{\prime} \in I_{n}=\left[t_{n-1}, t_{n}\right]$, then

$$
\begin{gathered}
f_{0}(t)-x_{n}=\frac{\left(x_{n}-x_{n-1}\right)}{h}\left(t-t_{n}\right) \\
f_{0}\left(t^{\prime}\right)-x_{n}=\frac{\left(x_{n}-x_{n-1}\right)}{h}\left(t^{\prime}-t_{n}\right)
\end{gathered}
$$

Then:

$$
\begin{gathered}
f_{0}(t)-f_{0}\left(t^{\prime}\right)=\frac{\left(x_{n}-x_{n-1}\right)}{h}\left(t-t^{\prime}\right) \\
\left|f_{0}(t)-f_{0}\left(t^{\prime}\right)\right| \leq \frac{\left|x_{n}-x_{n-1}\right|}{h} \frac{\pi}{m} \leq \frac{N \Delta_{x}}{2 m}
\end{gathered}
$$

and the inequality (31) is fulfilled.

If $t, t^{\prime}$ belong to consecutive intervals, $t<t_{n}<t^{\prime}$, applying (32)

$$
\left|f_{0}(t)-f_{0}\left(t^{\prime}\right)\right| \leq\left|f_{0}(t)-f_{0}\left(t_{n}\right)\right|+\left|f_{0}\left(t_{n}\right)-f_{0}\left(t^{\prime}\right)\right| \leq \frac{N \Delta_{x}}{m}
$$


Proposition 5.4. Let $f_{0}$ be as in Proposition 5.2 (continuous, piecewise linear and $f_{0} \in \mathcal{C}(2 \pi)$ ). Then, for $m$ sufficiently large,

$$
\left\|f_{0}-S_{m} f_{0}\right\|_{\infty} \leq \frac{(4+\log m) N \Delta_{x}}{m+1}
$$

where $\Delta_{x}=\max _{1 \leq n \leq N}\left\{\left|x_{n}-x_{n-1}\right|\right\}$.

Proof. Let $S^{*}$ be the trigonometric polynomial of degree $m$ such that

$$
d_{m}^{*}\left(f_{0}\right)=\left\|f_{0}-S^{*}\right\|_{\infty}=\inf \left\{\left\|f_{0}-S\right\|_{\infty} ; S \in \tau_{m}\right\}
$$

where

$$
\tau_{m}=<\{1, \sin t, \cos t, \sin 2 t, \cos 2 t, \ldots, \sin m t, \cos m t\}>
$$

then

$$
\begin{gathered}
\left\|f_{0}-S_{m} f_{0}\right\|_{\infty} \leq\left\|f_{0}-S^{*}\right\|_{\infty}+\left\|S^{*}-S_{m} f_{0}\right\|_{\infty}= \\
=\left\|f_{0}-S^{*}\right\|_{\infty}+\left\|S_{m} S^{*}-S_{m} f_{0}\right\|_{\infty} \leq(4+\log m) d_{m}^{*}\left(f_{0}\right)
\end{gathered}
$$

Theorems of Jackson [4] give upper bounds for $d_{m}^{*}\left(f_{0}\right)$. In particular, a Theorem states that for any function $f_{0} \in \mathcal{C}(2 \pi)$

$$
d_{m}^{*}\left(f_{0}\right) \leq w\left(\frac{\pi}{m+1}\right)
$$

Applying the Lemma 5.3 the result is obtained.

Theorem 5.5. Let $f$ be an AFIF respect to the data $\left\{\left(t_{n}, x_{n}\right)\right\}_{n=0}^{N}, f \in$ $\mathcal{C}(2 \pi)$. For $m$ sufficiently large

$$
\left\|f-\mathcal{S}_{m} f\right\|_{\infty} \leq(4+\log m)\left(\frac{2|\alpha|_{\infty}}{1-|\alpha|_{\infty}} X_{\max }+\frac{N \Delta_{x}}{m+1}\right)
$$

where $\mathcal{S}_{m} f$ is the finite sum of order $m, X_{\max }=\max _{0 \leq n \leq N}\left\{\left|x_{n}\right|\right\}$ and $\Delta_{x}=$ $\max _{1 \leq n \leq N}\left\{\left|x_{n}-x_{n-1}\right|\right\}$.

Proof. Let $f_{0} \in \mathcal{C}(2 \pi)$ be piecewise linear with vertices on the data $\left\{\left(t_{n}, x_{n}\right)\right\}_{n=0}^{N}$. One has

$$
\left\|f-S_{m} f\right\|_{\infty} \leq\left\|f-f_{0}\right\|_{\infty}+\left\|f_{0}-S_{m} f_{0}\right\|_{\infty}+\left\|S_{m} f_{0}-S_{m} f\right\|_{\infty}
$$

Applying the Propositions 5.1, 5.2 and 5.4:

$$
\left\|f-S_{m} f\right\|_{\infty} \leq(4+\log m)\left(\frac{2|\alpha|_{\infty}}{1-|\alpha|_{\infty}} X_{\max }+\frac{N \Delta_{x}}{m+1}\right)
$$


Theorem 5.6. Let $x(t)$ be a continuous periodic function sampled with step $h$ providing the data $\left\{\left(t_{n}, x_{n}\right)\right\}_{n=0}^{N}$, f the corresponding $A F I F$ and $\mathcal{S}_{m} f$ the finite Fourier sum of $f$ of order $m$. Then

$$
\left\|x-S_{m} f\right\|_{\infty} \leq w(h)+(5+\log m) \frac{2|\alpha|_{\infty}}{1-|\alpha|_{\infty}}\|x\|_{\infty}+(4+\log m) \frac{N \omega(h)}{m+1}
$$

where $w(\delta)$ is the modulus of continuity of $x$.

Proof. Let us consider

$$
\left\|x-S_{m} f\right\|_{\infty} \leq\left\|x-f_{0}\right\|_{\infty}+\left\|f_{0}-f\right\|_{\infty}+\left\|f-S_{m} f\right\|_{\infty}
$$

For the first adding let us consider the expression of the polygonal $f_{0}$ in $I_{n}=\left[t_{n-1}, t_{n}\right], \forall t \in I_{n}$,

$$
f_{0}(t)=x_{n-1}+\frac{x_{n}-x_{n-1}}{h}\left(t-t_{n-1}\right)
$$

to obtain that

$$
\left|x(t)-f_{0}(t)\right|=\left|\left(x(t)-x_{n-1}\right) \frac{t_{n}-t}{t_{n}-t_{n-1}}+\left(x(t)-x_{n}\right) \frac{t-t_{n-1}}{t_{n}-t_{n-1}}\right|
$$

then,

$$
\left|x(t)-f_{0}(t)\right| \leq \omega(h)
$$

and

$$
\left\|x-f_{0}\right\|_{\infty} \leq \omega(h)
$$

The second term is bounded by Proposition 5.1. For the third adding, let us consider Theorem 5.5 and the inequalities

$$
X_{\max }=\max _{0 \leq n \leq N}\left\{\left|x_{n}\right|\right\} \leq\|x\|_{\infty}
$$

and

$$
\Delta_{x}=\max _{1 \leq n \leq N}\left\{\left|x_{n}-x_{n-1}\right|\right\} \leq \omega(h)
$$

Consequence 5.7. If for each $m$ we choose a scaling vector $\alpha^{m}$ such that $\alpha^{m} \log m \rightarrow 0$ when $m \rightarrow \infty$, and assuming that $N \omega(h)$ is bounded, we obtain the convergence of the approximant $\mathcal{S}_{m} f$ to the original function when $h \rightarrow 0$ and $m \rightarrow \infty$. This fact assures a good approximation of $x$ whenever $h$ and $m$ are suitably chosen. 


\section{An EEG study}

The infantile EEG has been studied in connection with a large number of cognitive disabilities. To quote an example, an excessive slow EEG activity has been found associated with dyslexia in children. However, the utility of the visual analysis to evaluate this kind of patients has been questioned, due to the non-specificity of the findings. The work of the researchers in this area suggests that there exist significant differences in the recordings of these children, but more sophisticated tools are required. Our interest is based on the EEG description of homogeneous samples of children and its relations with cognitive activities, from different points of view. 
Our team has performed a numerical study of EEG recordings of two samples of children: a healthy control group and a set diagnosed with an Attention Deficit with Hyperactivity Disorder (ADHD). The clinical manifestations of the ADHD are characterised by a lack of attention, impulsive cognitive and behaviour styles and by an excessive motor activity. Its incidence is estimated between 3 and $5 \%$ of the school population and one or two children with deficient attention per classroom during the first school years could be observed. By a mere visual inspection of the EEG, no difference was observed in the patient group. Former ADHD EEG studies show the existence of an excessive slow activity, mainly localized in frontal area.

The children of control group were selected randomly by the teachers and belong to the same school groups than the children with ADHD. 19 children diagnosed with ADHD were chosen, with an average age of 9.3 and a standard deviation of 1.5. The sample was compared with a control group of 13 children with similar age (9.2) and standard deviation (1.3).

For every subject, the following signals were recorded: (i) an EEG of rest with closed eyes, (ii) an EEG during the execution of a test consisting in the recognition of a face different from the others, in series of three.

We analyzed six locations of the cortical surface following the 10-20 International System of Jasper: F3, F4, P3, P4, O1, O2. The sampling frequency was $128 \mathrm{~Hz}$. The computed neurophysiological indices were:

-Spectral parameters: Relative multiband amplitude. The spectral amplitude quantifies the rhythms of the EEG recordings which are familiar to the clinical diagnosis. We report here the results concerning alpha and beta bands, since these frequencies are typical of awake processes.

-Fractal dimension of the EEG trace computed by means of fractal interpolation of the signal [14]-[3].

The details and statistics can be read in [14] and [19]. The tables 1 and 2 collect the results for control group and the statistical significance of the recorded bioelectric changes. Beta amplitude and fractal dimension undergo a general increase during the execution of the test, with respect to closed eyes resting EEG all over the cortical surface, unlike the alpha band, where a similar decrease is recorded. The execution of the test produces significant differences in all the locations for spectral parameters. Beta frequency is predominant on the whole cortical surface during the test and the depression of alpha waves, in particular in the occipital area, is clearly observed. Regarding the fractal dimension, altough a wide increase is observed, the variations only reach significance in the right occipital position (O2). In the ADHD group, the results are qualitatively similar but there are more electrodes where the fractal dimension 


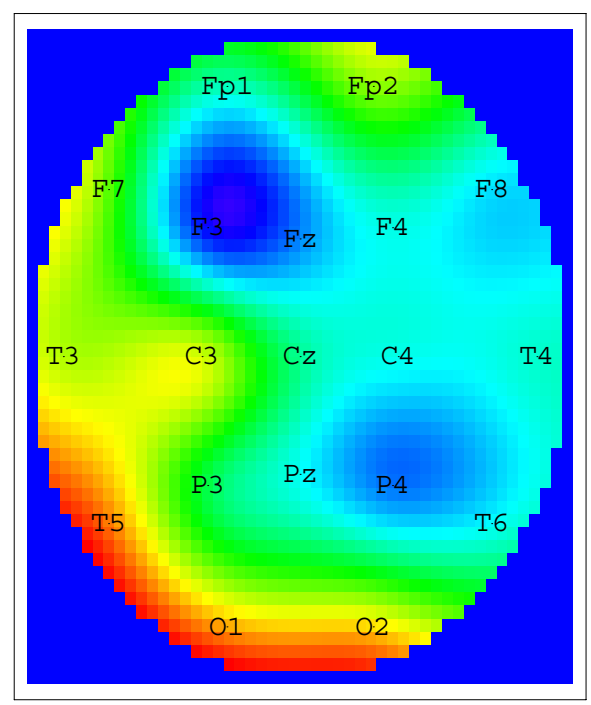

Figure 1: Brain mapping of fractal dimension of an individual at rest

is increased [14].

The sensory perceptions, cognitive tasks and motor activities change the model of blood flow in the brain cortex, revealing the localization of the areas involved in the respective processes. For instance, when an individual moves the fingers of the right hand, the cortical area corresponding to these fingers in the central cortex is activated. The execution of a task requiring an increase in the stress produces changes in the brain electric activity that are explicit in the desynchronization or depression of alpha rhythms [12], for instance. The decrease in alpha activity is considered an index of cortical activation.

In general, attention and sensory processes produce selective effects in the topographic distribution of EEG frequencies. The PET scan images prove that when a subject is undertaking intense or emotionally a task, an activation in the brain cortex is produced. The tests based on simple memory or deductive trials originate a total increase in the brain flow that may be $10 \%$ [8]. The variation is directly correlated with the effort developed by the individual in the execution of the trial. This stress is reflected here in the beta band, which undergoes significant changes all over the cortical surface, at the expense of alpha band. The changes in fractal dimensions are more selective, with percentages $5.9 \%, 3.5 \%$ and $2.9 \%$ in $\mathrm{O} 2$, $\mathrm{O} 1$ and $\mathrm{P} 4$ respectively for the control group. The variation is significant in the right occipital position (O2). In general, all the parameters that we have used show a higher activation of the right occipital area (O2) respect to the left occipital (O1) in the execution of the visual test.

Luria [9] reports that, for the injuries of the right occipital area, an im- 


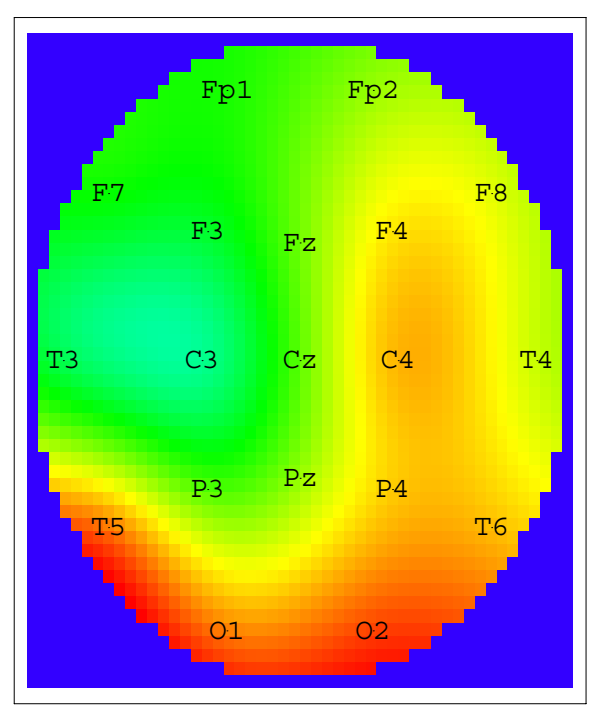

Figure 2: Brain mapping of fractal dimension of an individual executing the test of face recognition

portant characteristic is the symptom of prosopagnosia. This kind of patients can not remeber very familiar faces and frequently they only recognize a friend by his voice and not by the visual impression. The recognition of faces is an activity ruled by brain regions located in the inferior sides of temporal and occipital lobes [6]. The wound that destroys this area alters the capacity of recognising the persons by their facial features.

Without will of extracting medical conclusions, which would deserve a wider study, our results strengthen the hypothesis of Lutzenberger et al. [11] pointing out that the variations in the fractal dimensions are related to local metabolic changes. Our experiments suggest that fractal dimensions are neurophysiological indices showing an increase of the neural activity in the stimulated areas. The representation of the parameters on different cortical zones proves that their distribution on the skull constitutes a useful bioelectrical map, due to the recording of the bioelectric patterns implied in different mental and cognitive processes (see Fig. 1 and Fig. 2).

\section{References}

[1] A. Babloyantz and A. Destexhe, Low-Dimensional Chaos in an Instance of Epilepsy, Proc. Natl. Acad. Sci. USA (Neurobiology), 83 (1986), 3513 $-3517$. 
[2] M.F. Barnsley, Fractal functions and interpolation, Constr. Approx., 2(4) (1986), 303-329.

[3] M.F. Barnsley, Fractals Everywhere, Academic Press, Inc., 1988.

[4] E.W. Cheney, Approximation Theory, AMS Chelsea Publ., 1966.

[5] T. Elbert, W. Lutzenberger, B. Rockstroh, P. Berg and R. Cohen, Physical aspects of the EEG in schizophrenics, Biol. Psychiatry, 32 (1992), $595-606$.

[6] N. Geschwind, In: The Brain, Scientific American, 1979.

[7] H.P. Hsu, Fourier Analysis, Simon \& Schuster. New York, 1970.

[8] N.A. Lassen, D.H. Ingvar, E. Skinhoj. In: The Brain, Scientific American, 1979, $193-204$.

[9] A.R. Luria, Higher Cortical Functions in Man, New York Plenum Press, 1966.

[10] W. Lutzenberger, N. Birbaumer, H. Flor, B. Rockstroh, and T. Elbert, Dimensional analysis of the human EEG and intelligence, Neuroscience Letters, 143 (1992), 10 - 14.

[11] W. Lutzenberger, T. Elbert, R. Birbaumer, W.J. Ray, and H. Schupp, The scalp distribuition of the fractal dimension of the EEG and its variations with mental tasks, Brain Topography, 5(1) (1992), 27 - 34.

[12] C.A. Mann, M.B. Stermann, D.A. Kaiser, Suppression of EEG rhythmic frequencies during somatomotor and visuomotor behavior, Int. J. Psychophysiol., 23 (1996), 1 - 7.

[13] M.A. Navascues and M.V. Sebastian, Generalization of Hermite functions by fractal interpolation, J. of Approx. Th., 131(1) (2004), 19 - 29.

[14] M.A. Navascues and M.V. Sebastian, Fitting curves by fractal interpolation: an aplication to the quantification of cognitive brain processes, In: Thinking in Patterns: Fractal and Related Phenomena in Nature, Novak M.M.(ed.), World Sci., 2004.

[15] N. Pradhan and P.K. Sadasivan, The nature of dominant Lyapunov exponent and attractor dimension curves of EEG in sleep, Comput. Biol. Med., 26(5) (1996), 419 - 428.

[16] J. Röschke, Neuropsychobiology, 25 (1992), 172 - 176. 
[17] M.V. Sebastian, M.A. Navascues et al., An alternative to correlation dimension for the quantification of bioelectric signal complexity, WSEAS Trans. on Biol. \& Biomed., 1(3) (2004), 357 - 362.

[18] C. Soize, Méthodes mathématiques en analyse du signal, Masson Paris, 1993.

[19] J.R. Valdizan, M.A. Navascues and M.V. Sebastian , Análisis espectral multibanda y descriptores normalizados de pendiente de electroencefalogramas infantiles, Rev. Neurol., 31(12) (2000), 1130 - 1136.

[20] M. Ziller, K. Frick, W.M. Herrmann, S. Kubicki, I. Spieweg and G. Winterer Neuropsychobiol., 32. 4 (1995), 45 - 51.

\section{Received: November 9, 2005}

